\title{
Prekarisierung im Lebenszusammenhang - Bewegung in den Geschlechterarrangements?
}

\author{
Christina Klenner/Svenia Pfahl \\ Sabine Neukirch/Dagmar Weßler-Poßberg
}

Prekäre Beschäftigung verändert vielfach den gesamten Lebenszusammenhang der Betroffenen. Auch Geschlechterarrangements können davon berührt werden. Infolge von Prekarisierungsprozessen können Haushaltskonstellationen entstehen, in denen Frauen zu Familienernährerinnen werden, d.h. sie versorgen die Familie überwiegend aus ihrem Einkommen. Der Beitrag geht der Frage nach, ob es in Haushalten, in denen Frauen die Familienernährerinnen sind, auch zu einer veränderten Aufteilung der Hausarbeit kommt.

\section{Einleitung}

In der Debatte um Prekarisierung ist die Verunsicherung thematisiert worden, die durch die Verbreitung von prekären Beschäftigungsverhältnissen entstanden ist (vgl. stellvertretend Castel/Dörre 2009). Mit Manske/Pühl erscheint uns, dass der Begriff „Prekarisierung“ sich als hilfreich erweist, derzeitige Restrukturierungsprozesse auf unterschiedlichen Ebenen zu beschreiben. Dabei geht es erstens um gesellschaftliche „Integrations- und Anomieprobleme“ (vgl. Castel 2000), zweitens darum, dass der gegenwärtige Wandel nicht nur ökonomisch bedingt, „sondern auch Produkt politischer Entscheidungen " ist, und drittens „können auch neue soziale Zumutungen an die Lebensführung der Einzelnen erfasst werden" (Manske/Pühl 2010, S. 10).

Die Frage, inwiefern die von prekären Beschäftigungsverhältnissen ausgehende Verunsicherung auch den Lebenszusammenhang der Betroffenen berührt, gerät erst allmählich in den Fokus sozialwissenschaftlicher Forschung (vgl. Völker 2008; Aulenbacher 2009; Götz/Lemberger 2009). Wahrgenommen wird inzwischen, dass die mit „Prekarität“ umschriebene Verunsicherung vielfältige Folgen hat und sich auch auf den Lebenszusammenhang auswirkt (Vogel 2004; Damitz 2007; Clement et al. 2009). Jedoch ist dies bislang noch nicht genauer untersucht worden. Auf der Basis von zwei explorativen Forschungsprojekten über den Lebenszusammenhang von Familienernährerinnen ${ }^{1}$ plädieren wir für eine Erweiterung des Prekaritätsbegriffs: Es gilt, Prekarität im Lebenszusammenhang zu betrachten.
In jüngster Zeit wurde zugleich darauf hingewiesen, dass nicht nur die Lebenschancen einengende Seite von Prekarisierungsprozessen analysiert werden müsse. Notwendig sei es vielmehr auch, gleichzeitig ihr mögliches Potenzial für neue lebensweltliche Lösungen in den Blick zu nehmen (Seifert 2009). Denn auch wenn man die Folgen der schwindenden Schutzfunktionen des Normalarbeitsverhältnisses nicht verharmlosen dürfe, gälte es doch zu erkennen, dass sich als Folge seiner Erosionstendenzen auch die Geschlechterverhältnisse, auf denen das Normalarbeitsverhältnis über Jahrzehnte hinweg basierte, verändern könnten. Hier könne es - wenngleich erzwungen und labil - zu Verschiebungen und Öffnungen kommen (Dölling 2005; Völker 2007; Fraser 2009; Manske/Pühl 2010). Wir haben empirisch untersucht, inwieweit tatsächlich Bewegung in die Geschlechterarrangements kommt, wenn Männer und Frauen von den Folgen prekärer Erwerbssituationen betroffen sind.

Zunächst aber zeigen wir, warum ein erweitertes Prekaritätsverständnis unter Einbezug des ganzen Lebenszusammenhangs sinnvoll ist (Abschnitt 2). Anschließend stellen wir das Design unserer empirischen Studien kurz vor (Ab-

\footnotetext{
1 Zwei parallel angelegte und auf einen Vergleich der Situation in West- und Ostdeutschland ausgelegte Forschungsprojekte ( „Flexible Familienernährerinnen “) wurden durch die Hans-BöcklerStiftung in den Jahren 2008-2010 finanziert: Unter der Leitung von Christina Klenner (WSI) führten Petra Drauschke bis 12/08, Svenja Pfahl und Katrin Menke (SowiTra, Berlin) ab 4/2009 empirische Untersuchungen in Ostdeutschland durch. Das zweite, auf Westdeutschland bezogene Teilprojekt wurde unter der Leitung von Ute Klammer (Universität Duisburg-Essen) von Sabine Neukirch und Dagmar Weßler-Poßberg durchgeführt.
}

schnitt 3). Abschnitt 4 geht, gestützt auf unsere Untersuchungsbefunde, dann der uns hier vor allem interessierenden Frage nach, ob sich Geschlechterarrangements verändern, wenn die Frau die Familienernährerin ist. Wir untersuchen dies anhand der häuslichen Arbeitsteilung der Partner. Abschnitt 5 zieht ein knappes Fazit.

\footnotetext{
Christina Klenner, Dr., ist Wissenschaftlerin im Wirtschafts- und Sozialwissenschaftlichen Institut (WSI) in der Hans-Böckler-Stiftung. Arbeitsschwerpunkte: Geschlechterverhältnisse, Frauenerwerbstätigkeit und WorkLife-Balance, Gleichstellung. e-mail: christina-klenner@boeckler.de Svenja Pfahl ist Soziologin und Geschäftsführerin des Instituts für sozialwissenschaftlichen Transfer (SowiTra) in Berlin. Arbeitsschwerpunkte: Arbeitszeit, Vereinbarkeit von Beruf und Familie, Geschlechterverhältnisse und Familie.

e-mail: svenja.pfahl@sowitra.de Sabine Neukirch ist wissenschaftliche Mitarbeiterin am Institut für Soziale Arbeit und Sozialpolitik der Universität DuisburgEssen. Arbeitsschwerpunkte: Vergleichende Wohlfahrtsstaatsforschung, Europäische Integration, Arbeitsmigration und Geschlechterverhältnisse. e-mail: sabine.neukirch@uni-due.de Dagmar Weßler-Poßberg ist wissenschaftliche Mitarbeiterin am Institut für Soziale Arbeit und Sozialpolitik der Universität Duisburg-Essen. Arbeitsschwerpunkte: WorkLife-Balance und betriebliche Familien- und Personalpolitik. e-mail: dagmar.wessler-possberg@uni-due.de
} 


\section{Ein erweitertes Verständ- nis von Prekarität}

\subsection{DIE PERSPEKTIVE DES LEBENS- ZUSAMMENHANGES}

In ihrer „alltäglichen Lebensführung“2 vollbringen Menschen permanent die „Arbeit des Alltags" (Jurczyk/Rerrich 1993). Hier fließen Handlungen in unterschiedlichen Lebensbereichen - Erwerbsarbeit, Fürsorgearbeit, Selbstsorge, soziale Beziehungen - im Idealfall aufeinander abgestimmt zusammen. Dabei gilt es - wie die feministische Kritik an einer Arbeitssoziologie, die lange auf die Erwerbsarbeit fixiert war, wiederholt betont hat -, das „Ganze“ der Arbeit in den Blick zu nehmen, also neben der Erwerbsarbeit auch die gesellschaftlich notwendige Sorgearbeit zu betrachten (Aulenbacher 2009; Nickel 2009).

Die meisten Menschen leben nicht allein im Haushalt. ${ }^{3}$ Dieser Aspekt spielt in wissenschaftlichen Analysen bisher vor allem dann eine Rolle, wenn untersucht wird, ob geringfügige oder Niedrigeinkommen als Folge prekärer Erwerbssituationen auch zu Einkommensprekarität führen oder im Haushaltskontext aufgefangen werden. Allerdings ist auf der Einkommensebene auch das Umgekehrte möglich, etwa dass Partnerinnen oder Partner von Arbeitslosen in Bedarfsgemeinschaften mit in Armut geraten (Kraemer 2008, S.83). Deswegen werden Haushaltseinkommen und Äquivalenzeinkommen insgesamt betrachtet (vgl. Bartelheimer in diesem Heft). Doch auch jenseits der Einkommensebene muss beachtet werden, dass auch die Lebensführung von Menschen in prekären Erwerbskonstellationen mit der der anderen Haushaltsmitglieder zu einer „familialen Lebensführung" verflochten ist (Jürgens 2001). Dabei ist die familiale Lebensführung unter anderem vom Geschlechterarrangement ${ }^{4}$ des Paares geprägt.

Eine entsprechende Erweiterung des Prekaritätsverständnisses ist einerseits erforderlich, weil aus der Perspektive des Lebenszusammenhangs der Blick auf andere bzw. zusätzliche Aspekte der Erwerbsarbeit gelenkt wird, die verunsichernd wirken. Damit rücken zeitliche Aspekte, aber auch Belastungs-, Sicherheits- sowie Planungsaspekte von Erwerbsarbeit stärker in den
Blick, die für die Koordinierung einer familialen Lebensführung unverzichtbar sind. Prekarität in der Erwerbsarbeit kann bei dieser Betrachtung nicht nur in atypischen Beschäftigungsverhältnissen und Niedriglohnbeschäftigung, sondern auch innerhalb der „Normalarbeit“" zu beobachten sein (vgl. Jürgens in diesem Heft).

Andererseits ermöglicht die erweiterte Perspektive den Blick auf das Zusammenspiel unterschiedlicher Dimensionen, die sich zu einer prekären Gesamtlage verdichten können. Allein die mangelnde Passfähigkeit verschiedener Elemente des Lebenszusammenhangs kann eine prekäre Lage hervorbringen. Jede einzelne Aktivität - Care-Aufgaben, oft für mehrere Kinder, berufliche Verpflichtungen, Pflege, Wohnen, Verkehr, Gesundheitspflege - weist stets raum-zeitliche Dimensionen auf, die untereinander passfähig sein müssen. Daneben sind soziale, organisatorische, finanzielle u.a. Aspekte zu berücksichtigen. So greift die prekäre Erwerbsarbeitssituation wegen ihrer Dominanz häufig auf andere Lebensbereiche über. Zum Beispiel kann die berufliche Mobilität eines Partners, die durch stets nur befristete Beschäftigungen bedingt ist, dazu zwingen, Betreuungsarrangements permanent neu zu justieren.

Die Perspektive des Lebenszusammenhangs ermöglicht es, die Mehrdimensionalität von Verunsicherung (Kraemer 2008) zu erfassen, welche nicht nur die verschiedenen in der familialen Lebensführung miteinander verbundenen Personen, sondern auch unterschiedliche Lebensbereiche betreffen kann. Unsicher und fragil können auch der Fürsorgebereich (Care), die Selbstsorge, die Einbindung in das soziale Leben, die Entwicklungschancen der Kinder sowie die finanzielle Situation des Haushalts einschließlich der Möglichkeit der Altersvorsorge werden.

Quellen für Prekarität im Lebenszusammenhang können somit sowohl prekäre Beschäftigungsformen als auch Arbeitsbedingungen innerhalb scheinbarer Normalarbeit sein. Zudem kann sich Prekarität im Lebenszusammenhang auch als Folge sozialstaatlicher Aktivierungsstrategien einstellen, da diese Rekommodifizierungsprozesse mit sich bringen, deren Bewältigung Individuen überfordern können. Weiterhin können auch individuelle Schicksalsschläge wie Krankheiten oder Verbrechen im Lebenszusammenhang prekarisierend wirken.

\subsection{KENNZEICHEN VON PREKARITÄT IM LEBENSZUSAMMENHANG}

Von einer abschließenden Klärung dessen, was Prekarität ist, was sie ausmacht, kann derzeit keine Rede sein. Es handelt sich bei dem Begriff um eine Art „Platzhalter, der Momentaufnahmen einer spezifischen, im Verschwinden begriffenen historischen Realität festzuhalten beansprucht (...)“ (Damitz 2007, S. 78).

Der Kern von Prekarität ist, dass die Betroffenen sich Planungs- und Gestaltungsunsicherheiten gegenübersehen (Kraemer 2008). Sie sind „verwundbar“, ihre Lebenslage ist instabil. Prekarität ist „nicht identisch mit vollständiger Ausgrenzung aus dem Erwerbssystem, absoluter Armut, totaler sozialer Isolation und erzwungener politischer Apathie. Vielmehr handelt es sich um eine relationale Kategorie, deren Aussagekraft wesentlich von der Definition gesellschaftlicher Normalitätsstandards abhängt“ (Brinkmann et al. 2006, S. 17). Der Prekaritätsbegriff hat seine Wurzeln auch im „prekarium“, d.h. der Gewährung eines Rechts auf eine Bitte hin (Reinprecht 2008). Das heißt, die von prekären Verhältnissen Betroffenen können sich nicht auf ausreichende rechtliche Ansprüche stützen. In der Folge ist ihre Handlungsfähigkeit eingeschränkt. Zusätzlich wurde die subjektive Seite des Prekaritätsempfindens herausgearbeitet, die sich von der objektiven Lage unterscheiden kann. Und schließlich wurde auch bereits darauf aufmerksam gemacht, dass es für die Beurteilung von Prekarität relevant ist, ihre „Verzeitlichung“ (Kraemer 2008) zu beachten, also danach $\mathrm{zu}$ fragen, ob prekäre Erwerbs- und/oder Lebensverhältnisse vorübergehender Art sind oder sich umgekehrt biografisch verfestigen.

Um den Prekaritätsbegriff nicht durch zu breite Verwendung letztlich zu entleeren, sollte ein auf den Lebenszusammenhang erweitertes Prekaritätsverständnis den inhaltlichen Gehalt des Prekaritäts-

2 Der Begriff „alltägliche Lebensführung“ umschreibt "das System der alltäglichen Tätigkeiten der Person" mit seinen zeitlichen, räumlichen, sozialen, sinnhaften, geschlechtlichen u.a. Strukturdimensionen (Voß 2001, S. 203ff.).

3 Unter allen Haushalten sind 31 \% Single-Haushalte (Brehmer et al. 2010), auf der Personenebene ist der Anteil noch deutlich niedriger.

4 Völker sieht "Geschlechterarrangements" sowohl als relativ verfestigte (aber veränderbare) Strukturen und Institutionen als auch als praktizierte Lebens- und Arbeitsweisen (Völker 2008, S. 289). 
begriffes bewahren und nicht jede ungünstige Lage als prekär bezeichnen. Als zentrale Charakteristika von Prekarität im Lebenszusammenhang sehen wir daher an:

- Planungs- und Gestaltungsunsicherheiten des Lebens,

- Unterschreiten historisch gegebener Normalitätsstandards,

- Lebensgestaltung auf Widerruf sowie - eingeschränkte Handlungsalternativen, Ohnmachts- und Diskriminierungserfahrungen.

Diese Charakteristika finden sich, wie unsere empirischen Befunde deutlich zeigen, in unterschiedlichen Kombinationen in verunsicherten Lebenszusammenhängen wieder. ${ }^{5}$ Bezogen auf die familiale Lebensführung gibt es zahlreiche Planungs- und Gestaltungsunsicherheiten, die beispielsweise daraus resultieren, dass tagtäglich Arbeitszeiten und Fürsorgezeiten miteinander ausbalanciert werden müssen. Aus der empirischen Forschung wissen wir, dass gefundene Arrangements vielfach „prekäre Balancen“ (Eberling et al. 2004) sind, die sogleich „auf der Kippe“ stehen, wenn etwa eine Person aus dem Betreuungsnetzwerk ausfällt oder das Kind (z.B. durch chronische Erkrankung) einen erhöhten Betreuungsbedarf hat.

Prekarität im Lebenszusammenhang ist eine relationale Kategorie. Als prekär sehen wir beispielsweise an, wenn gesellschaftliche Normalitätsstandards ${ }^{6}$ bezüglich der Betreuung und Zuwendung gegenüber Kindern unterschritten werden. Wenn z.B. hiesige Standards, wie die ausreichende Betreuung von Kleinkindern, die Unterstützung der schulischen Leistungen bei älteren Kindern und/oder der Anspruch auf Vorsorgeuntersuchungen und Therapien der Kinder durch die Eltern aus zeitlichen, finanziellen oder organisatorischen Gründen nicht gewährleistet werden können, ist dies ein Indiz für Prekarität im Lebenszusammenhang.

Mitunter fehlt es auch an gesicherten Ansatzpunkten (wie beispielsweise rechtlichen Ansprüchen oder am Vermögen, auf solche zurückzugreifen), um vorhandene Prekaritätsdimensionen aufzulösen. Zentrale Seiten der Lebensgestaltung sind abhängig vom Entgegenkommen anderer, das jederzeit widerrufbar ist. Nicht selten stehen Eltern gegenüber Ämtern oder Kindereinrichtungen als Bittsteller da, weil sie keinen gesicherten Anspruch haben. Die Balance ihres Familienalltags hängt „am seidenen Faden“ ungesicherter Zugeständnisse von Arbeitgebern oder anderen, sodass sie ihr Leben nur auf Widerruf gestalten können. Prekär kann die Lebensführung von Eltern auch werden, wenn sie gesetzlich verbürgte Ansprüche wie Elternzeit, Teilzeit oder eine Freistellung bei Erkrankung der Kinder nicht in Anspruch nehmen können, weil sie sich angesichts der Arbeitsmarktlage gezwungen sehen, einem hohen Verfügbarkeitsanspruch des Arbeitgebers nachzugeben.

Prekarität im Lebenszusammenhang zeigt sich auch daran, dass sich Betroffene in Konstellationen mit widersprüchlichen Handlungsanforderungen gefangen sehen. Ohnmachts- und Diskriminierungserfahrungen sowie erlebte Rechtlosigkeit, gegen die sich auch Interessenvertretungsinstanzen als machtlos erweisen, lassen sie an ihrer Selbstwirksamkeit zweifeln. In der Folge werden auch die privaten Lebensarrangements fragil.?

In der deutschen Prekaritätsdebatte bisher unterbelichtet blieb das multiple Jobholding (Vogel 2004), teilweise in $\mathrm{Zu}$ sammenhang mit der Aufnahme irregulärer Beschäftigungen, die unter Umständen auch wegen der Fürsorgeverpflichtungen alternativlos erscheinen. Beschäftigungsformen mit nicht-existenzsichernden Einkommen, die meist eine deutlich geschlechtsspezifische Ausprägung aufweisen, machen es notwendig, in mehreren Jobs zu arbeiten (Mangan 2000).

Zusammengefasst: Wir definieren Prekarität im Lebenszusammenhang als eine Gefährdungs- und Unsicherheitslage, die neben der Fragilität der individuellen auch die familiale Lebensführung betreffen kann und die eingeschränkte Handlungsfähigkeit oder gar einen Verlust an Zukunft - möglicherweise für mehrere Personen - mit sich bringt. Es geht in dieser sozialwissenschaftlichen Betrachtung von Prekarisierung weder darum, die individuelle Betroffenheit von schweren Schicksalsschlägen (Krankheit, Tod, Trennung) an sich, noch das prinzipiell Prekäre des Seins (Butler 2009 zit. in Hark/Völker 2010, S. 43) oder die prinzipiell gegebene Unsicherheit des Lebens zu thematisieren. Vielmehr soll der Blick dafür geschärft werden, dass individuelle Schicksalsschläge heute unter Umständen wieder mehr als in vergangenen Jahrzehnten, in denen die soziale Unsicherheit von "Strukturen kollektiver Absicherung" gebändigt war (Castel 2009), dazu führen können, dass Einzelne in prekäre Lagen kommen. Es geht um den Verlust von bereits errungenen Sicherheiten. Damit hat der Prekaritätsbegriff auch eine sozialkritische Dimension.

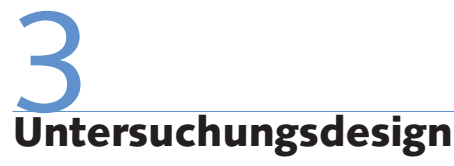

Wie bereits erwähnt, basieren unsere hier vorgestellten Ausführungen auf den Ergebnissen aus zwei empirischen Studien (vgl. Fußnote 1), in deren Rahmen wir quantitative Auswertungen (des Sozio-oekonomischen Panels) und qualitative Forschungsmethoden (90 problemzentrierte Interviews mit erwerbstätigen Frauen aus West-/Ostdeutschland) kombiniert haben.

Wir haben Familienernährerinnen als Untersuchungsgruppe ausgewählt, weil sie eine geradezu modellhafte Verkörperung des „adult worker model“ darstellen, das die Europäische Beschäftigungsstrategie als sozialpolitisches Leitmodell etabliert hat. Dieses Leitbild zielt darauf, dass jeder und jede den notwendigen Lebensunterhalt durch eigene Erwerbsarbeit sichern soll (Lewis 2004). Da Familienernährerinnen, wenn sie Kinder haben, die Verantwortung für Einkommenserwerb und Fürsorgearbeit zu bewältigen haben - was bei klassischer Arbeitsteilung üblicherweise auf zwei Partner mit mehr oder minder polarisierten Geschlechterrollen verteilt wird-, nehmen wir an, dass Widersprüche des adult-worker-model bei dieser Gruppe früher und ausgeprägter zutage treten als bei anderen Gruppen. Zwar wurde mit dem Zuwachs an FamilienernährerinnenHaushalten ein emanzipatorisches Potenzial assoziiert (Bittman et al. 2003), es wird jedoch auch davon ausgegangen, dass sich für einige Gruppen von Familienernährerinnen dieser Status mit erheblichen Brüchen verbindet. ${ }^{8}$ Da sich die weitere

5 Die empirischen Belege können hier aus Platzgründen nicht dargestellt werden (vgl. dazu Klammer et al. 2011; Klenner et al. 2011).

6 Normalitätsstandards sind in einem demokratischen Aushandlungsprozess zu definieren und können sich im Lauf der Zeit und zwischen sozialen Gruppen unterscheiden (vgl. Bartelheimer in diesem Heft).

7 Für empirische Belege siehe die beiden Projektberichte (Klammer et al. 2011; Klenner et al. 2011).

8 Es handelt sich um Studien mit explorativem Charakter, sodass Aussagen über die Reichweite der Befunde nicht getroffen werden können. 
Erwerbsintegration von Frauen häufig in Form atypischer Beschäftigung vollzieht, während Männer einer zunehmenden Fragilisierung ihrer Erwerbsintegration unterworfen sind (Klammer/Daly 2003; Cranford et al. 2003), wurden für beide Studien flexibel bzw. atypisch beschäftigte Familienernährerinnen befragt.

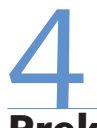

\section{Prekarität und häusliche Arbeitsteilung}

Bezüglich der durch Prekarisierung ausgelösten Veränderungsimpulse auf die Geschlechterverhältnisse gibt es unterschiedliche Auffassungen (vgl. Abschnitt 1): Ein Diskurs thematisiert die Chance auf Öffnungen im Geschlechterverhältnis (Völker 2008; Knuth 2006), ein anderer erwartet eher Re-Traditionalisierungen ${ }^{9}$ in den Geschlechterarrangements als Prekarisierungsfolge (Dörre 2007).

Unsere Forschungsergebnisse zeigen zunächst, dass in nahezu jedem zehnten Paarhaushalt ${ }^{10}$ in Deutschland die Frau die Haupteinkommensbezieherin ist. Der Anteil ist in Ostdeutschland höher als in Westdeutschland. Dass Frauen in diesem Umfang Familienernährerinnen sind, kann auf der einen Seite das Ergebnis sowohl der anwachsenden, zunehmend kontinuierlichen und qualifizierten Erwerbstätigkeit von Frauen mit entsprechenden Einkommen sein. Auf der anderen Seite aber kann diese Konstellation aus der Prekarisierung männlicher Erwerbsarbeit, also u.a. aus Arbeitslosigkeit und Niedriglöhnen der männlichen Partner im Haushalt resultieren. Eine multivariate Analyse des WSI zeigt, dass der zweite Entwicklungsstrang stärker wirksam ist als der erste (Brehmer et al. 2010).

Wir konzentrieren uns im Folgenden auf die Frage, ob sich in Familienernährerinnen-Haushalten der letztgenannten Konstellation (Prekarisierung männlicher Erwerbsarbeit) auf Paarebene auch an den Geschlechterarrangements etwas ändert und betrachten dafür speziell die häusliche Arbeitsteilung.

\subsection{THEORETISCHE ANNAHMEN ZUR HAUSARBEITSTEILUNG}

Gemäß der Theorien der Haushaltsökonomie (Becker) und des Time-AvailabilityAnsatzes (Coverman) ergibt sich die Teilung der Hausarbeit aus dem rationalen Prozess der Entscheidung auf der Grundlage der potenziellen Verdienstmöglichkeiten, der Stärken beider Partner und ihrer jeweiligen zeitlichen Ressourcen zur Maximierung des Nutzens für den Haushalt. Die Ressourcentheorie und die Austauschtheorie hingegen nehmen die ökonomischen Ressourcen beider Partner wie Bildung, berufliche Position und Einkommen hinsichtlich einer stärkeren Aushandlungsmacht in den Blick. Während dies in der Ressourcentheorie scheinbar geschlechtsneutral der Partner mit den höchsten Einkommenschancen ist, der die Hausarbeit an den anderen (in der Regel die Frau) delegieren kann (vgl. zusammenfassend Geist 2005; Wengler et al. 2008), geht die Austauschtheorie davon aus, dass ein Partner aufgrund seines (oder ihres) höheren Einkommens mehr Aushandlungsmacht hat und den anderen Partner/die Partnerin dazu bewegen kann, mehr Hausarbeit zu leisten. Haushaltsarbeit wird im Austausch mit finanziellem Unterhalt geleistet, was eine unsymmetrische Relation ist, die den Weg für Ausbeutung ebnet (Brines 1994, S. 656).

Auf der Basis solcher Theorieansätze zur Erklärung der Hausarbeitsteilung kann man annehmen, dass die häusliche Arbeitsteilung neu verhandelt und vielleicht im Ergebnis anders gestaltet wird, wenn Frauen die Familienernährerinnen sind. Offensichtlich sind in dieser Konstellation ihre Verdienstmöglichkeiten besser, zugleich dürfte der Familienernährerinnen-Status mit einer stärkeren zeitlichen Belastung der Frau mit Erwerbsarbeit einhergehen. Auch die Ressourcen zwischen den Partnern sind ungleich verteilt, wenn sie deutlich mehr verdient, und der Mann, als finanziell Abhängiger, müsste in ein Mehr an unbezahlter Arbeit einwilligen.

In der bisherigen Forschung zu Familienernährerinnen dominiert allerdings der Befund, dass auch in Haushalten, in denen die Frau für das relevante Haushaltseinkommen verantwortlich ist, es ganz überwiegend bei einer ungleichen, geschlechtsspezifischen Arbeitsteilung bleibt (Stamp 1985; Brines 1994; Bittman et al. 2003; Tichenor 2005; Drago et al. 2005). Zur Erklärung dieser familialen Arbeitsteilung wurden, angelehnt an die Geschlechterrollentheorie, Modelle entwickelt, die die Kategorie "Geschlecht“ als zentral für die Persistenz der Aufgabenteilung erachten. Entsprechend der kulturellen Traditionen übernehmen „weiblich“ sozialisierte und auch fernerhin mit weiblichen Rollenerwartungen konfrontierte Frauen mehr Hausarbeit, um ihre Geschlechtsidentität zu wahren (Geist 2007 zit. in Wengler et al. 2008). Ebenso schützen Männer ihre Geschlechterrollenidentität, indem sie angesichts des Verlustes der männlichen Ernährerrolle nicht zudem noch weiblich konnotierte Tätigkeiten im Haushalt übernehmen (England 2006, zit. in Wengler et al. 2008, S. 16). Das Paar entwickelt somit gemeinsam im Alltag Strategien, die die Devianz des gelebten Modells, also die Abweichung vom „üblichen Muster“, durch traditionelle Elemente abmildern sollen (Brines 1994; Tichenor 2005; Meisenbach 2009). Pappenheim/Graves (2005) hingegen fanden auch Gegenbeispiele. Eine umfangreichere männliche Partizipation bzw. Hauptverantwortlichkeit des männlichen Partners für die Hausarbeit wurde dort beobachtet, wo beide Partner egalitär orientierte Einstellungen aufwiesen (vgl. dazu auch Drago et al. 2005; Duncan 2005).

\subsection{EMPIRISCHE BEFUNDE: MODIFIKATION UND PERSISTENZ DER HAUSARBEITSTEILUNG}

Männer, die in Haushalten mit einer Frau als Familienernährerin leben, leisten im Durchschnitt mehr Familienarbeit als Männer, die selber die Familienernährer sind. Das zeigen unsere Auswertungen auf der Grundlage des Sozio-oekonomischen Panels (SOEP). ${ }^{11}$ Allerdings übernehmen dieser Analyse zufolge gleichwohl die Familienernährerinnen - wie alle Frauen - durchschnittlich mehr Hausarbeit als ihre Partner. Die folgenden zwei Beispiele, die für typische Konstellationen unserer qualitativen Analysen stehen, bestätigen einerseits dieses Grundmuster (Fall 1), zeigen aber auch die Persistenz traditioneller Formen der häuslichen Arbeitsteilung selbst unter geänderten Rahmenbedingungen (Fall 2).

9 Gemeint ist eine Rückkehr zum „fordistischen Geschlechterregime" (Young 1998).

10 Haushalte mit mindestens einer Erwerbsperson, das heißt, reine Rentnerhaushalte wurden ausgeklammert.

11 Berechnungen durch Tanja Schmidt 2011 im Auftrag des WSI. 
FALL 1: PRAGMATISCHE UMORIENTIERUNG MINDERT PREKARITÄT

Bei Familie W. in Sachsen-Anhalt, einem Paar in der „erzwungenen Notgemeinschaft" ${ }^{\text {"12 }}$, hat sich die häusliche Arbeitsteilung hin zu mehr Familienarbeit des Mannes verschoben, seitdem er langzeitarbeitslos ist. Doch auch wenn er inzwischen deutlich mehr Haus- und Fürsorgearbeit übernimmt, leistet Frau W. immer noch den größeren Anteil. Bereits vor der Veränderung beteiligte sich der Ehemann regelmäßig an der Kinderbetreuung, da die Schichtarbeit von Frau W. Arbeitszeiten bis 23.20 Uhr einschloss. Voraussetzung war, dass Herr W. als Heizungsinstallateur vergleichsweise familienkompatible Arbeitszeiten hatte. Die Ehefrau, die trotz zweier Berufsabschlüsse keine adäquate Beschäftigung finden konnte, arbeitet unterqualifiziert in einer Großwäscherei in Vollzeit. Ursprünglich aus Russland stammend, stand ihre eigene Erwerbstätigkeit für Frau W. nie zur Disposition. Dennoch vertraten beide Partner eine eher traditionelle Geschlechterrollenorientierung: Es war selbstverständlich, dass eigentlich die Mutter trotz Vollzeittätigkeit hauptverantwortlich für Haushalt und Kind ist. Seitdem Herr W. vor längerer Zeit arbeitslos geworden ist, befindet sich diese Gewissheit im Wandel. Er übernimmt nun einen größeren Teil der Kinderbetreuung und der Hausarbeit. Er hat gelernt, Wäsche zu waschen und zu bügeln. Insbesondere als Pflegeleistungen für seine Eltern notwendig werden, übernimmt er auch hier weitere Aufgaben. Diese pragmatische Umorientierung hat einen Wandel in den geschlechterbezogenen Vorstellungen nach sich gezogen. Sie ist aber auch durch die sozialpolitische Rahmung mit vorangetrieben worden: Seit der Arbeitslosigkeit des Vaters hat der vierjährige Sohn nur noch Anspruch auf eine Halbtagsbetreuung in der Kindertagesstätte. Zugleich hat Frau W. aktiven Anteil an der veränderten Familienarbeitsteilung. Sie ist die Familienernährerin und fordert unter Verweis auf ihre Erwerbstätigkeit und seine Arbeitslosigkeit seine Mitarbeit ein.

Die Veränderung der Hausarbeitsteilung entlastet Frau W. und lindert die Prekarität im Lebenszusammenhang des Paares, die sich aus dem Zusammenspiel von Einkommensarmut, Langzeitarbeitslosigkeit, Belastungskumulation und eingeschränkten sozialpolitischen Ansprü- chen ergeben hat. Für Herrn W. geht mit der Linderung der Prekarität allerdings eine sich verfestigende Verunsicherung seiner Erwerbssituation einher, da er keine mit dem aktuellen Familienarrangement kompatible Stelle mehr finden kann. Alle infrage kommenden Jobs verlangen mehrtägige Abwesenheit von zu Hause. Da die Stelle seiner Frau für den Lebensunterhalt unabdingbar ist, kann er nur eine Stelle annehmen, die mit der Kinderbetreuung vereinbar wäre. So sehr das pragmatisch modernisierte Geschlechterarrangement hier zur Abmilderung der prekären familialen Lebenssituation beiträgt, es reicht nicht, um erwerbsbedingte Prekarität für beide Partner abzuwenden.

\section{FALL 2: TRADITIONELLE ARBEITS- TEILUNG VERSCHÄRFT PREKARITÄT}

Frau S., gelernte Bürokauffrau in Nordrhein-Westfalen, nahm nur deshalb nach Jahren der familienbedingten Erwerbsunterbrechung eine bezahlte Tätigkeit auf, weil ihr Mann nach einer Erkrankung langzeitarbeitslos wurde. Bereits die Rolle der Zuverdienerin ist kaum mit ihren Vorstellungen über eine Paarbeziehung und über die „richtige“ elterliche Aufgabenteilung vereinbar; die der Familienernährerin ist es noch viel weniger: In ihren Augen sollte die Mutter für die Kinder sorgen. Ihre Re-Integration in den Arbeitsmarkt gestaltete sich von Anfang an prekär: Frau S. konnte keinen Anschluss an ihre Erstqualifikation gegenüber der Arbeitsagentur erwirken und musste ungewollt eine Call-Center-Schulung durchlaufen. Dem folgte eine Vielzahl von Beschäftigungen in Call-Centern im Nachtdienst (zugunsten der Betreuung ihrer drei Söhne am Tage), die von arbeitsrechtlichen Verstößen, hohem Leistungsdruck und fehlenden Möglichkeiten kollektiver Gegenwehr geprägt waren. Selbst in diesem Zeitraum blieb ihr langzeitarbeitsloser Mann so gut wie vollständig von Haus- und Fürsorgeleistungen entlastet.

In dem Bemühen, künftig der Vermittlung in ein Call-Center durch die Arbeitsagentur zu entgehen, wurde Frau S. notgedrungen zur Mehrfacherwerbstätigen (multiple Jobholder) in zumeist befristeten, niedrig entlohnten und häufig irregulären Aushilfstätigkeiten, die - aufgrund des Pflegebedarfs der Schwiegereltern ebenfalls im Schichtdienst ausgeübt wurden. Insgesamt ging Frau S. in zehn Jah- ren zwölf verschiedenen Beschäftigungen nach. Ihre prekären Erwerbsbedingungen und die fortgesetzte Verantwortlichkeit für die Haus- und Fürsorgearbeit der erweiterten Familie blieben nicht folgenlos: Die Ehe ist zerrüttet, ihre Belastungen übertrugen sich auf die Kinder und mündeten in gesundheitliche Beeinträchtigungen und schließlich einen Zusammenbruch der Familienernährerin. Zwar ist Herr S. heute (de-qualifiziert) wieder vollzeitbeschäftigt, jedoch erzwingt die finanziell prekäre Situation der gesamten Familie (auch der mittlerweile erwerbstätigen Söhne) nach wie vor die berufliche Tätigkeit auch von Frau S. Sie hat ihre Erwerbssituation weder finanziell noch qualifikatorisch verbessern oder stabilisieren können und antizipiert eine gesundheitlich und finanziell fragile Zukunft für die ganze Familie. Wohlfahrtsstaatliche Fehlregulierung verschränkt sich im Fall der Familie S. mit einer fragilen Erwerbssituation und einer Persistenz geschlechtsspezifischer Arbeitsteilung, die den Geschlechterleitbildern beider Partner entspricht, zu einem insgesamt prekären Lebenszusammenhang.

Fall 1 scheint die theoretischen Annahmen der Haushaltsökonomie und des Time-Availibility-Ansatzes zu bestätigen, dass Paare entsprechend der Verdienstmöglichkeiten und zeitlichen Ressourcen von Mann und Frau Erwerbstätigkeit und Familienarbeit zum Nutzen der Familie teilen. Damit zeigt der Fall, wie Dölling (2005), Völker (2008) und Manske/Pühl (2010) annehmen, dass es unter prekären Bedingungen einen Anstoß zu Neuverhandlungen und Öffnungen im Geschlechterarrangement geben kann. Die Umverteilung der Haus- und Fürsorgearbeit ist Folge der erzwungenermaßen beschränkten Verdienstmöglichkeiten des Mannes und der zeitlichen Belastung der Frau als Hauptverdienerin. Die Umorientierung bei Familie W. knüpft aber an eine bereits vorher gegebene gewisse Aufgeschlossenheit gegenüber einer Veränderung der Geschlechterrollen an. Genau dies ist im

\footnotetext{
12 Wir konnten fünf Typen von Familienernährerinnen-Paaren identifizieren. Der Typ der "erzwungenen Notgemeinschaft" ist dadurch gekennzeichnet, dass die Frau den Familienernährerinnen-Status unfreiwillig/unbeabsichtigt aus der Not heraus übernimmt, häufig auch Alleinverdienerin ist, weil der Mann arbeitslos, erwerbsunfähig oder prekär beschäftigt ist. Zugleich ist eine angespannte Einkommenssituation des Haushaltes typisch (Klenner et al. 2011).
} 
Fall 2 nicht gegeben. Beide Partner lassen sich von einer traditionell polarisierten Geschlechterrollenvorstellung leiten und haben stets eine geschlechtsspezifische Arbeitsteilung praktiziert. Dies ändern sie auch nicht, als die Frau die Haupternährerinnen-Rolle übernehmen muss. Fall 2 bestätigt, dass „Geschlecht“ eine entscheidende Rolle bei der Aufteilung der Hausarbeit spielt. Beide Partner wollen eine noch weitergehende Abweichung von den Geschlechterrollenleitbildern, wie sie mit der Übernahme von Hausarbeit durch den Mann verbunden wäre, vermeiden. Dies bestätigt die Devianz-Theorie von Brines.

In beiden Fällen leisten die Frauen trotz ihrer Erwerbstätigkeit und Familienernährerinnen-Rolle mehr Hausarbeit als ihre Partner. Selbst dort, wo es zu einer Verschiebung der Arbeitsteilung kommt, kommt es nicht zu einer egalitären Aufteilung und erst recht nicht zu einem vollständigen Rollentausch. Auch dies bestätigt, dass trotz Umorientierung die Geschlechterrollen weiterhin die Teilung der unbezahlten Arbeit beeinflussen.

Wir haben zwei Faktoren identifizieren können, die Einfluss darauf haben, ob die unbezahlte Arbeit zumindest teilweise umverteilt wird. Dies sind, erstens, die Arbeitsmarktposition des Mannes, und damit die Ursachen für das Entstehen der weiblichen Familienernährer-Konstellation, und, zweitens, die Geschlechterrollenleitbilder beider Partner. Sind die Männer nur mit geringem Einkommen oder gar nicht stabil in den Arbeitsmarkt integriert, können Frauen sich auf ihre höheren Erwerbsarbeitsbelastungen berufen und es kann zu einer Neuverhandlung der häuslichen Arbeitsteilung kommen. Zum anderen kommt es bei Paaren mit stärker egalitär ausgerichteten Geschlechterrollenleitbildern eher zu nicht-traditionalen Lösungen, während ein Festhalten an traditionellen Vorstellungen zur Abwertung neuer Rollenverteilungen oder Resignation hinsichtlich der Veränderungsmöglichkeiten beiträgt.

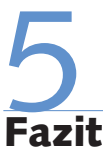

Prekarität im Lebenszusammenhang, die sich unter anderem in ausgeprägten Vereinbarkeitskonflikten, fragilen Betreuungslösungen und gesundheitlichen Problemen ausdrückt, kann Individuen in umfassender Weise fordern, kreativ neue Handlungsstrategien zu entwickeln - aber manchmal auch überfordern.
Betroffene mobilisieren alle möglichen Ressourcen, um ihre Lebensführung abzusichern und stellen dabei unter Umständen auch traditionelle arbeitsteilige Strukturen zwischen den Geschlechtern zur Disposition. Das gilt für Familienernährerinnen in Bezug auf die Arbeitsteilung bei der Einkommenserwirtschaftung. Das kann aber auch für die Umverteilung der Familienarbeit gelten. Geschlechterarrangements können sich somit als Ressource erweisen, Folgen prekärer Arbeit zu mildern und eine Prekarität im Lebenszusammenhang zu verhindern oder wenigstens zu lindern. Kommt es nicht zu Neuverhandlungen der häuslichen Arbeitsteilung, kann die Prekarität im Lebenszusammenhang hingegen noch verschärft werden.

Die Erweiterung des Prekaritätsverständnisses auf den Lebenszusammenhang ist nicht nur von wissenschaftlicher Bedeutung, sondern sie hat auch eine gesellschaftspolitische Dimension. Die Ergebnisse unserer Studien belegen einen drängenden politischen Handlungsbedarf. Umso mehr sind Wissenschaft und Politik gefordert, neben den Erwerbsarbeitsbedingungen verstärkt auch den familialen Lebenszusammenhang in den Blick zu nehmen.

\section{LITERATUR}

Aulenbacher, B. (2009): Die soziale Frage neu gestellt. Gesellschaftsanalysen der Prekarisierungs- und Geschlechterforschung, in: Castel, R./Dörre, K. (Hrsg.): Prekarität, Abstieg, Ausgrenzung. Die soziale Frage am Beginn des 21. Jahrhunderts, Frankfurt a. M., S. 65-77 Bittman, M./England, P./Sayer, L./Folbre, N./Matheson, G. (2003): When Does Gender Trump Money? Bargaining and Time in Household Work, in: American Journal of Sociology 109 (1), S. 186-214

Brehmer, W./Klenner, C./Klammer, U. (2010): Wenn Frauen das Geld verdienen - eine empirische Annäherung an das Phänomen der "Familienernährerin“, WSI-Diskussionspapier (170), Düsseldorf

Brines, J. (1994): Economic Dependency, Gender and the Division of Labor at Home, in: American Journal of Sociology 100 (3), S. 652-688 Brinkmann, U./Dörre, K./Röbenack, S. (2006): Prekäre Arbeit. Ursachen, Ausmaß, soziale Folgen und subjektive Verarbeitungsformen unsicherer Beschäftigungsverhältnisse, Friedrich-Ebert-Stiftung, Bonn Castel, R. (2000): Die Metamorphosen der sozialen Frage. Eine Chronik der Lohnarbeit, Edition discours, Bd. 13, Konstanz
Castel, R. (2009): Die Wiederkehr der sozialen Unsicherheit, in: Castel, R./Dörre, K. (Hrsg.): Prekarität, Abstieg, Ausgrenzung. Die soziale Frage am Beginn des 21. Jahrhunderts, Frankfurt a. M., S. 21-34 Castel, R./Dörre, K. (Hrsg.) (2009): Prekarität, Abstieg, Ausgrenzung. Die soziale Frage am Beginn des 21. Jahrhunderts, Frankfurt a. M. Clement, W./Mathieu, S./Prus, S./Uckardesler, E. (2009): Precarious lives in the new economy - Comparative intersectional analysis, in: Vosko, L./MacDonald, M./Campbell, I. (Hrsg.): Gender and the Contours of Precarious Employment, London, S. 240-255

Cranford, C.J./Vosko, L. F./Zukewich, N. (2003): The Gender of Precarious Employment in Canada, in: Industrial Relations 58 (3), S. 454-482 Damitz, R.M. (2007): Prekarität. Genealogie einer Problemdiagnose, in: Mittelweg 36 (4), S. 67-86

Dölling, I. (2005): Ostdeutsche Geschlechterarrangements in Zeiten des neoliberalen Gesellschaftsumbaus, in: Schäfer, E. (Hrsg.): Irritation Ostdeutschland. Geschlechterverhältnisse in Deutschland seit der Wende, Münster, S. 16-34 
Dörre, K. (2007): Prekarisierung und Geschlecht. Ein Versuch über unsichere Beschäftigung und männliche Herrschaft in nachfordistischen Arbeitsgesellschaften, in: Aulenbacher, B. (Hrsg.): Arbeit und Geschlecht im Umbruch der modernen Gesellschaft, Wiesbaden, S. 285-301

Drago, R./Black, D./Wooden, M. (2005): Female breadwinner families. Their existence, persistence and sources, in: Journal of Sociology 41 (4), S. 343-362

Duncan, S. (2005): Mothering, class and rationality, in: The Sociological Review 53 (1), S. 50-76

Eberling, M./Hielscher, V./Hildebrandt, E./Jürgens, K. (2004): Prekäre Balancen. Flexible Arbeitszeiten zwischen betrieblicher Regulierung und individuellen Ansprüchen, Berlin

Fraser, N. (2009): Feminismus, Kapitalismus und die List der Geschichte, in: Blätter für deutsche und internationale Politik 54 (8), S. 43-57

Geist, C. (2005): The Welfare State and the Home: Regime Differences in Domestic Division of Labour, in: European Sociological Review 21 (1), S. $23-41$

Götz, I./Lemberger, B. (Hrsg.) (2009): Prekär arbeiten, prekär leben. Kulturwissenschaftliche Perspektiven auf ein gesellschaftliches Phänomen, Frankfurt a. M.

Hark, S./Völker, S. (2010): Feministische Perspektiven auf Prekarisierung: Ein "Aufstand auf der Ebene der Ontologie", in: Manske, A./ Pühl, K. (Hrsg.): Prekarisierung zwischen Anomie und Normalisierung. Geschlechtertheoretische Bestimmungen, Münster, S. 26-47 Jürgens, K. (2001): Familiale Lebensführung. Familienleben als alltägliche Verschränkung individueller Lebensführungen, in: Voß, G. G./ Weihrich, M.(Hrsg.): Tagaus, tagein. Neue Beiträge zur Soziologie alltäglicher Lebensführung, München, S. 33-60

Jurczyk, K./Rerrich, M. S. (Hrsg.) (1993): Die Arbeit des Alltags. Beiträge zu einer Soziologie der alltäglichen Lebensführung, Freiburg i. B. Klammer, U./Daly, M.(2003): Die Beteiligung von Frauen an europäischen Arbeitsmärkten, in: Gerhard, U./Knijn, T./Weckwert, A. (Hrsg.): Erwerbstätige Mütter. Ein europäischer Vergleich, München, S. $193-217$

Klammer, U./Neukirch, S./Wessler-Possberg, D. (2011): Flexible Familienernährerinnen. Eine Studie zur Entwicklung von Arbeitsbedingungen und Geschlechterverhältnissen in Westdeutschland, unveröff. Projektbericht der Hans-Böckler-Stiftung, Düsseldorf

Klenner, C./Menke, K./Pfahl, S. (2011): Flexible Familienernährerinnen. Prekarität im Lebenszusammenhang ostdeutscher Frauen?, unveröffentlichter Projektbericht der Hans-Böckler-Stiftung, Düsseldorf Knuth, M. (2006): „Hartz IV" - die unbegriffene Reform, in: Sozialer Fortschritt 55 (7), S. 160-168

Kraemer, K. (2008): Prekarität - was ist das?, in: Arbeit 17 (2), S. $77-90$

Lewis, J. (2004): Auf dem Weg zur "Zwei-Erwerbstätigen“-Familie, in: Leitner, S./Ostner, I./Schratzenstaller, M. (Hrsg.): Wohlfahrtsstaat und Geschlechterverhältnis im Umbruch. Was kommt nach dem Ernährermodell? Wiesbaden, S. 62-84
Mangan, J. (2000): Workers Without Traditional Employment: An International Study of Non-Standard Work, Cheltenham/Northampton Manske, A./Pühl, K. (Hrsg.) (2010): Prekarisierung zwischen Anomie und Normalisierung. Geschlechtertheoretische Bestimmungen, Forum Frauen- und Geschlechterforschung, Bd. 28, Münster

Meisenbach, R. (2009): The Female Breadwinner: Phenomenological Experience and Gendered Identity in Work/Family Spaces, in: Sex Roles $62(1-2)$, S. $2-19$

Nickel, H. M. (2009): Zum 20. Jahrestag des Mauerfalls - Eine Bilanz aus ostdeutscher feministischer Perspektive, in: Femina politica 18 (2), S. $107-111$

Pappenheim, H./Graves, G. (2005): Bringing home the bacon. Making the marriage work when she makes more money, New York

Reinprecht, C. (2008): Prekarisierung und Re-Feudalisierung sozialer Ungleichheit, in: Kurswechsel 23 (1), S. 13-23

Seifert, M. (2009): Prekarisierung der Arbeits- und Lebenswelt - Kulturwissenschaftliche Reflexionen zu Karriere und Potenzial eines Interpretationsansatzes, in: Götz, I./Lemberger, B. (Hrsg.): Prekär arbeiten, prekär leben. Kulturwissenschaftliche Perspektiven auf ein gesellschaftliches Phänomen, Frankfurt a. M., S. 31-53

Stamp, P. (1985): Research Note: Balance of Financial Power in Marriage. An Explanatory Study of Breadwinning Wives, in: Sociological Review 33 (3), S. 546-557

Tichenor, V. (2005): Maintaining Men's Dominance. Negotiating Identity and Power. When She Earns More, in: Sex Roles 53 (3/4), S. $191-205$

Trappe, H./Sørensen, A. (2005): Economic Relations between Women and Their Partners: An East-West-German Comparison after Reunification, DIW-Discussion Paper (544), Berlin

Völker, S. (2007): Prekäre Transformationen - herausgeforderte Lebensführungen, in: Bock, U./Dölling, I./Krais, B. (Hrsg.): Prekäre Transformationen. Pierre Bourdieus Soziologie der Praxis und ihre Herausforderungen für die Frauen- und Geschlechterforschung, Göttingen, S. 176-194 Völker, S. (2008): Entsicherte Verhältnisse - (Un)Möglichkeiten fürsorglicher Praxis, in: Berliner Journal für Soziologie 18 (2), S. $282-306$

Vogel, B. (2004): Neue Ungleichheiten im Wohlfahrtsstaat - Die politische Ordnung sozialer Verwundbarkeit und prekären Wohlstands, in: Zeitschrift für Sozialreform 50 (1-2), S. 174-188

Voß, G. G. (2001): Der eigene und der fremde Alltag, in: Voß, G. G./ Weihrich (Hrsg.): Tagaus, tagein. Neue Beiträge zur Soziologie alltäglicher Lebensführung, München, S. 203-217

Wengler, A./Trappe, H./Schmitt, C. (2008): Partnerschaftliche Arbeitsteilung und Elternschaft. Analysen zur Aufteilung der Hausarbeit und Elternaufgaben auf Basis des Generation and Gender Survey, Bundesinstitut für Bevölkerungsforschung, Materialien zur Bevölkerungswissenschaft (127), Wiesbaden

Young, B. (1998): Genderregime und Staat in der globalen NetzwerkÖkonomie, in: PROKLA 111, 28 (2), S. 175-198 